\title{
"Characterization of ion beam irradiated 304 stainless steel utilizing nanoindentation and Laue microdiffraction"
}

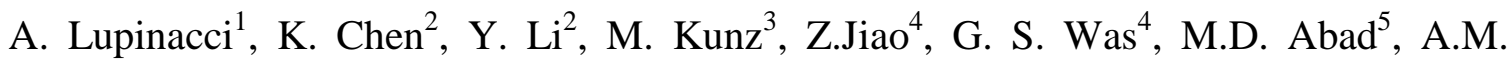 \\ Minor $^{1,6}$, P. Hosemann ${ }^{5 *}$ \\ ${ }^{1}$ Department of Materials Science and Engineering, University of California, Berkeley, CA \\ ${ }^{2}$ Center for Advancing Materials Performance from the Nanoscale (CAMP-Nano), State Key Laboratory \\ for Mechanical Behavior of Materials, Xi'an Jiaotong University, Xi'an 710049, P.R. China \\ ${ }^{3}$ Advanced Light Source, Lawrence Berkeley National Laboratory, Berkeley, CA \\ ${ }^{4}$ Department of Nuclear Engineering, University of Michigan, Ann Arbor, MI \\ ${ }^{5}$ Department of Nuclear Engineering, University of California, Berkeley, CA \\ ${ }^{6}$ National Center for Electron Microscopy, The Molecular Foundry, Lawrence Berkeley National \\ Laboratory, Berkeley, CA
}

*Corresponding author: 4169 Etcheverry hall; phone: 510-717-5752;

email:Peterh@berkeley.edu

\begin{abstract}
Characterizing irradiation damage in materials utilized in light water reactors is critical for both material development and application reliability. Here we use both nanoindentation and Laue micro diffraction to characterize both the mechanical response and microstructure evolution due to irradiation. Two different irradiation conditions were considered in 304 stainless steel: 1dpa and $10 \mathrm{dpa}$. In addition, an annealed condition of the $10 \mathrm{dpa}$ specimen for 1 hour at $500{ }^{\circ} \mathrm{C}$ was evaluated. Nanoindentation revealed an increase in hardness due to irradiation and also revealed that hardness saturated in the 10 dpa case. Broadening using Laue micro diffraction peaks indicates a significant lattice strain in the irradiated area that is in good agreement with both the SRIM calculations and the nanoindentation results.
\end{abstract}




\section{Introduction}

Radiation damage in materials is a significant concern in advanced and conventional nuclear reactors, spallation sources, isotope production facilities and fusion technology applications. Embrittlement and environment interaction are key issues during lifetime extension or performance predictions for reactors ${ }^{1,2,3,4}$. Of particular interest is the effect of irradiation in stainless steels such as 304 and 316L due to their widespread use as structural materials in light water reactors ${ }^{5}$. However, when subjected to irradiation, austenitic steels harden due to the formation of defect clusters that act as obstacles to dislocation motion under an applied stress ${ }^{6}$. Depending on the material, irradiation temperature, and stacking fault energy of the system, these defect clusters can manifest themselves as stacking fault tetrahedra (SFT) and dislocation loops ${ }^{7-8}$. Furthermore, irradiation induced hardening results in a decrease in ductility, which has been well characterized in metals 9 . Therefore it is important to thoroughly characterize and understand both the defects that are introduced through irradiation and the corresponding change in mechanical properties.

Since reactor components become radioactive in service, especially stainless steels due to the high nickel content, ion beam irradiations are considered as a surrogate for reactor irradiations ${ }^{10}$. In addition ion beam damage has a significant higher damage rate than neutron irradiation and therefore high doses can be achieved in a matter of days rather than years. However, medium energy $(2 \mathrm{MeV}$ ) light ions $(\mathrm{H})$ have a limited penetration depth. Higher ion beam energies are possible but reduce the displacement per atom (dpa) rate while resulting in greater sample activation. One of the benefits of using a low energy proton beam is that it can be used to investigate radiation damage without highly activating the specimen. However one of the limitations to using a lower energy source is the limited penetration depth, therefore necessitating the use of nanoscale post irradiation characterization methods such as nanoindentation ${ }^{11-12}$ and synchrotron radiation based X-ray Laue microdiffraction $(\mu \mathrm{XRD})^{13}$. In this work, these methods are applied to investigate the change in mechanical properties as a result of ion beam irradiation and mapping of the microstructure induced by the ion beam irradiation.

In this study 304 stainless steel (304SS) was studied in two different dose conditions: 1 dpa, $10 \mathrm{dpa}$. Additionally, the $10 \mathrm{dpa}$ sample was also annealed to determine whether the radiation damage can be annealed out at $500{ }^{\circ} \mathrm{C}$. This paper reports the nanoindentation experiments of both configurations as well as the corresponding $\mu \mathrm{XRD}$ of the irradiated region. The purpose of this study is to characterize the hardening induced by ion irradiation and correlate that with the defect density estimated by $\mu$ XRD.

\section{Experimental}

The irradiation experiment was conducted using $2 \mathrm{MeV}$ protons in a Tandetron accelerator at the Michigan Ion Beam Laboratory (MIBL). Two specimens of 304SS were irradiated to 1 and $10 \mathrm{dpa}$, respectively, at $360^{\circ} \mathrm{C}$ with a dose rate of $\sim 8 \times 10^{-6} \mathrm{dpa} / \mathrm{s}$ based on the SRIM calculation ${ }^{14}$ (Full cascade option used with a displacement energy of $40 \mathrm{eV}$ for $\mathrm{Fe}, \mathrm{Cr}$ and $\mathrm{Ni}$ ). The equivalent doses would be 0.5 and $5 \mathrm{dpa}$, respectively, if 
the K-P option is used in SRIM. The sample temperature was monitored using a twodimensional (2D) thermal imager and the variation was kept within $\pm 10{ }^{\circ} \mathrm{C}$ during the course of irradiation. A detailed description of the proton irradiation procedure was published elsewhere ${ }^{15}$. The annealed specimen was subjected to $500{ }^{\circ} \mathrm{C}$ for 1 hour in a vacuum furnace. Figure 1 summarizes the dose profile calculated using SRIM where the $\mathrm{x}$ axis represents the penetration depth of the ion beam in cross section.

\subsection{Sample preparation for nanoindentation}

Both the 1 dpa and 10 dpa samples were polished in cross section with respect to the irradiated surface. During polishing each sample was mounted directly next to a thin piece of steel to prevent deformation and rounding near the edge of the specimen. The samples were planarized using $\mathrm{SiC}$ grinding paper with water as a lubricant and were then polished with $0.3 \mu \mathrm{m}, 0.1 \mu \mathrm{m}$ alumina polishing solutions, and $0.05 \mu \mathrm{m}$ colloidal silica polishing solution.

\subsection{Nanoindentation measurements}

Nanoindentation measurements were performed at the Nuclear Materials Laboratory at the University of California, Berkeley on the Micro Materials NanoTest ${ }^{\mathrm{TM}}$. The nanoindenter was calibrated against fused silica before each indentation run to allow for cross comparison between samples and indenters ${ }^{16}$. Indents were $200 \mathrm{~nm}$ deep and a minimum of $4 \mu \mathrm{m}$ apart from each other to ensure no interaction of the plastic zone around the indents. An array of $10 \times 8$ indents was set near the irradiated edge and an array of $8 \times 8$ indents was set on the opposite side where no beam had hit the surface. Comparing the irradiated with the unirradiated edge of the sample ensures that there were no edge effects, and observed differences can be attributed to ion beam irradiation. The indent field in the irradiated region were tilted towards the edge intentionally to increase the depth resolution and potentially resolve the stopping peak, which is shown in Figure 2 17. The indents were all performed in depth-control mode with a loading and unloading rate of $2 \mathrm{mN} / \mathrm{s}$ and a dwell time of 5 seconds. The hardness was obtained by the OliverPharr method ${ }^{18}$. In support of the nanoindentation measurements, an indentation size effect study was performed on the surface of a 10 dpa specimen in both the control and irradiated region. Indents were measured at various depths. A set of three indents was performed at each unique depth. The size effect study was performed on a Hysitron Triboindenter using a Berkovich tip.

Each indentation field was examined using scanning electron microscopy to verify the location of each indent with respect to the sample edge. The data reported (hardness vs. depth from the irradiated surface) reflects the SEM measurements.

\section{$2.3 \mu \mathrm{XRD}$ study}

Synchrotron radiation based Laue microdiffraction experiments were conducted at Beamline 12.3.2 of the Advanced Light Source (ALS) at Lawrence Berkeley National 
Laboratory ${ }^{19}$. A polychromatic X-ray beam $(5-24 \mathrm{keV})$ was focused to $\sim 1 \times 1 \mu \mathrm{m}^{2}$ by a pair of Kirkpatrick-Baez (KB) mirrors. A schematic of the Laue diffraction setup can be found in the study presented by M. Kunz et al. ${ }^{20}$ The samples were mounted on a high resolution $x-y$ scan stage with its polished cross-section facing up, and then tilted $45^{\circ}$ relative to the incident X-ray beam. For each sample, a fast fluorescence scan was conducted to position the sample edge so as to ensure that all Laue diffraction scans covered the region from the sample edge to about $100-150 \mu \mathrm{m}$ towards the sample matrix. In this study the diffraction scanning step size was $2 \mu \mathrm{m}$ and the exposure time at each position was $1 \mathrm{~s}$. Diffraction patterns were recorded in reflection mode with a $2 \mathrm{D}$ Pilatus detector mounted at $90^{\circ}$ to the incoming X-ray, approximately $140 \mathrm{~mm}$ from the probe spot. The detector has $1043 \times 981$ pixels and each pixel is about $170 \mu \mathrm{m}^{2}$ in size. Calibrations for sample-to-detector distance, center channel position and tilt of detector were performed by indexing a Laue diffraction pattern from a low-strain sharp diffraction pattern within the sample far from the edge using an in-house developed analysis software package XMAS ${ }^{21}$. Reflection peak shapes were fitted with a 2D Gaussian function so that the peak position and width were determined with a precision of about 0.1 pixels, providing an angular resolution of $\sim 0.01^{\circ}$.

\section{Results}

\subsection{Nanoindentation results}

Figure 3 shows the hardness profile as a function of irradiation depth in cross section indention as described above. Starting from the original sample surface the hardness change as a function of distance from the edge is shown. Both sides (irradiated and not irradiated) are displayed for comparison reasons in one graph. The 1 dpa sample hardness profile is closely related to the calculated dpa profile (Figure 1) with higher hardness at the end of the stopping region and less hardness at the near surface areas as it is displayed in Figure 3. It was found that the hardness of the bulk was approximately $(3.23 \pm .36)$ $\mathrm{GPa}$ whereas in the irradiated region of the $10 \mathrm{dpa}$ sample the hardness saturated at approximately $(5.58 \pm .22) \mathrm{GPa}$ as shown in Figure 4. Maximum hardness in the $10 \mathrm{dpa}$ sample was achieved at $19.7 \mu \mathrm{m}$ from the irradiated surface with a corresponding hardness of $6.05 \mathrm{GPa}$, whereas in the $1 \mathrm{dpa}$ irradiated region the maximum hardness was reached at $20.8 \mu \mathrm{m}$ from the irradiated surface (at the stopping peak) with a corresponding hardness of $6.13 \mathrm{GPa}$.

However, as can be seen in Figure 4, the irradiated region in the 10 dpa sample exhibited a marked increase in hardness. Hardness in the irradiated region doubled with respect to the control region (from 3.2 to $5.5 \mathrm{GPa}$ ). Interestingly, no dose related profile (increase of hardness with indentation depth) was observed, suggesting that the maximum possible hardness increase was reached at $10 \mathrm{dpa}$ in the flat part of the region and no additional hardening due to higher damage in the stopping peak was observed. Essentially it appeared that the amount of possible hardening is saturated at this dose. Figure 2 shows an SEM micrograph of the corresponding indent field for the $10 \mathrm{dpa}$ specimen. 
The transition region can be found at a depth of $\sim 21 \mu \mathrm{m}$ depth which is slightly deeper than the calculated depth by SRIM. One possible reason for this discrepancy can be found in the fact that the volume tested by the indenter is in fact larger than the position of the indent. A $200 \mathrm{~nm}$ deep indent is $\sim 1 \mu \mathrm{m}$ wide and samples are $\sim 2-2.5 \mu \mathrm{m}$ wide area in $\mathrm{x}$ and $\mathrm{y}$ making spacing between indents of at least $4 \mu \mathrm{m}$ necessary.

An indentation size effect study was also performed on both the irradiated region of the 10 dpa sample as well as an unirradiated specimen, as can be seen in Figure 5. This study was only performed at the $10 \mathrm{dpa}$ sample due to the fact that the 1dpa sample showed such a strong hardness gradient in cross section that de-convoluting the size effect from the dose profile and therefore hardening profile is challenging. The size effect is quantified using the Nix and Gao plot in the insert in Figure 5 and discussed further in the discussion section. We also want to emphasize that the size effect and actual cross section hardness testing was performed on two different systems (Hysitron and Micromaterials) as well as with different indenters. The area functions produced by the two systems agree rather well.

In addition to the irradiated 10 dpa sample, an annealed 10 dpa sample was also evaluated. Figure 6 summarizes the nanoindentation results for the annealed sample. Compared to Figure 4, the irradiated region showed a small decrease in the measured hardness; however, as seen in Figure 6, the annealing did not completely remove much of the damage that was induced by the ion irradiation.

\subsection{Laue Diffraction results}

By indexing the $\mu \mathrm{XRD}$ patterns assuming face-centered cubic iron phase, the crystal orientation at all scanning positions is obtained, and the out-of-plane orientation map of the 10 dpa irradiated sample near the irradiation edge is demonstrated in Figure 7a. Colors indicate lattice orientations at each scan spot as given by the color scale (inset). White spots in the figure suggest that there are no or only very low-intensity diffraction patterns recorded, either because the beam is out of the sample (the upper part of the figure) or as a result of defects/impurities (within the sample). Twins are clearly visible in the orientation map and the twin plane is the $\{111\}$ plane. For over $80 \%$ of the scanned area the $\{100\}$ or $\{110\}$ plane normals are parallel to the out of plane normal. The grain size is much larger than $40 \mu \mathrm{m}$, so that it is probable that the array of nanoindentation ( $32 \mu \mathrm{m} \times 28 \mu \mathrm{m}$ in area) was performed within a single crystal grain.

Diffraction peak broadening is correlated to the defect density in a specimen ${ }^{22,23}$. The peak width distribution in the scanned 10 dpa irradiated sample is plotted in Figure $7 \mathrm{~b}$. The peak width here is defined as the average FWHM, in the unit of degrees, of all recorded reflections in each Laue diffraction pattern. A sharp boundary is observed in this map, indicating that the diffraction peaks taken from the region within approximately $20 \mu \mathrm{m}$ from the sample surface are significantly broadened compared to those beyond the proton range. Typical Laue patterns from the matrix and surface of the specimen are demonstrated in Figure 7c and d, respectively. Although these two patterns were taken on a crystal in which the orientation stays nearly unchanged, the shape of the peaks from 
both patterns is clearly different. The peaks in Figure 7c are only slightly wider than the ones taken on defect-free thin Si films, while in Figure $7 \mathrm{~d}$ all the peaks are broadened. In order to reveal the mechanism that the peak broadening is rooted from, quantitative peak shape analysis is performed on one of the typical broadened peaks, the peak $1 \overline{1} 5$ in Figure $7 \mathrm{~d}$. First of all, the peak is re-plotted in the Bragg-azimuthal angle $(2 \theta-\chi)$ space, as shown in Figure 7e. Then the intensity of the diffraction peak is scrutinized in both $2 \theta$ and $\chi$ directions along the dashed lines drawn in Figure 7e, and fitted with Gaussian function, respectively (displayed in Figure $7 \mathrm{f}$ ). It is found that although appearing elongated anisotropically on the detector image in Figure $7 \mathrm{~d}$, the diffraction peak shape becomes more isotropic in the angular $2 \theta-\chi$ space. The FWHM in the $2 \theta$ and $\chi$ directions is $0.63^{\circ}$ and $0.59^{\circ}$, respectively. The isotropic broadening indicates statistically stored dislocations (SSD), with equal number of positive and negative Burger's vectors rather than an ordered array of unpaired geometrically necessary dislocations (GNDs), which leads to linearly streaked reflections ${ }^{24,25,26}$.

The peak width distribution maps of the unirradiated, $1 \mathrm{dpa}$, and annealed $10 \mathrm{dpa}$ samples are shown in the same color scale in Figure 8a-c, respectively. Not surprisingly, the unirradiated specimen gives sharp and uniform diffraction peaks over the entire scanned area. The matrix of both the $1 \mathrm{dpa}$ and the annealed 10 dpa samples diffract similarly sharp as the unirradiated one, while the reflections from the surface of the irradiated ones are broadened. Although the affected depth shows little dependence of the irradiation dose and/or thermal treatment, the damage is more severe in the 10 dpa sample than in the $1 \mathrm{dpa}$ one, even after annealing for $1 \mathrm{hr}$ at $500{ }^{\circ} \mathrm{C}$. However, comparing Figure 8c with Figure $7 \mathrm{~b}$, it is concluded that the damage is slightly recovered by the annealing, evidenced by less broadened peaks (less red-orange but more green spots are found in Figure $8 \mathrm{c}$ than in $7 \mathrm{~b}$ ).

\section{Discussion}

As shown in the results it was found that the hardness increases due to 1dpa irradiation following the damage profile calculated by SRIM, and the hardness at the 10dpa sample plateaus over the entire radiation damaged region despite the fact that the SRIM profile predicts an increasing dose of one order of magnitude from the flat part of the region to the peak. This strongly suggests that past a dose of $10 \mathrm{dpa}$, no further hardening can be detected which suggests that the material is saturated in radiation induced defects. Similar effects are seen on reactor irradiated samples where no further change is observed on $304 \mathrm{SS}$ irradiated beyond $10 \mathrm{dpa}$ at $330-375^{\circ} \mathrm{C}^{27}$. Spallation source irradiated material or lower temperature $275{ }^{\circ} \mathrm{C}$ reactor irradiation shows this saturation at doses as low as 3-4 $\mathrm{dpa}^{10,28}$. In this experiment it can be seen that the 1dpa sample shows a similar hardness at the end of range at approximately $20 \mu \mathrm{m}$ where the dose is $\sim 10 \mathrm{dpa}$ as well. Therefore we propose that saturation occurs by $10 \mathrm{dpa}$ in our study. While in our study it is still difficult to state the exact dose at which hardening occurs due to the lack of intermediate dose samples in between where also a plateau would be expected at a specific depth, it appears that our data agrees well with $\sim 330{ }^{\circ} \mathrm{C}$ irradiated materials from reactors. However, it can also be stated that the effect of saturation is not unique to this irradiation 
but has been observed in the other studies mentioned above and agrees well with the literature.

In order to correlate the data shown here with macroscopic mechanical tests it is necessary to account for the size effect observed with nanoindentation as first reported by Schulz and Hanemann ${ }^{29}$ and widely studied on a number of materials $s^{30,31,32,33}$. In our study it was found that the irradiated material shows a significantly smaller size effect than the unirradiated material. Utilizing the Nix and Gao equation one can calculate the parameters $\mathrm{H}_{0}$ and $\mathrm{h}^{*}$ which accounts for the size effect. The Nix and Gao model provides a description of the depth dependence of hardness. $\mathrm{H}_{0}$ is the hardness that arises from statistically stored dislocations alone and characterizes the hardness of infinite depth, in the absence of any geometrically necessary dislocations, and $h^{*}$ is a length that characterizes the depth dependence of the hardness. It was found that for the irradiated material $\mathrm{H}_{0}$ and $\mathrm{h}^{*}$ are 4.75 and 75.41, respectively. The unirradiated material $\mathrm{H}_{0}$ and $\mathrm{h}^{*}$ are 1.91 and 319.2, respectively. Comparison with literature values (smaller $\mathrm{h}^{*}$ and higher $\mathrm{H}_{0}$ numbers) reveals that the irradiated material shows a trend similar to cold worked materials where the deformed material shows a smaller size effect $^{34}$. This is based on the fact that both cold working and irradiation cause an increase in dislocations and therefore a decreased size effect as discussed $\mathrm{in}^{35}$ Taking into account the fact that typical Vickers hardness is measured in $\mathrm{kg} / \mathrm{mm}^{2}$ and the slightly different geometry one comes to the conclusion that between Vickers hardness and nano hardness the equation 1 applies:
$\mathrm{H}_{\mathrm{V}}=0.0945 \cdot \mathrm{H}_{\mathrm{Berk}}$
Eq. 1

where the indenter geometry is considered $\left[{ }^{35}\right]$. In $\left[{ }^{36}\right]$ the factor 0.0937 is used in the same equation based on empirical values. We utilize the geometrical relationship based on A.C.Fisher Cripps, and calculate a micro hardness of $448 \mathrm{H}_{\mathrm{V}}$ on the irradiated material and $180 \mathrm{H}_{\mathrm{V}}$ on the unirradiated material. Jiao et al. ${ }^{37}$ found the unirradiated hardness from microhardness measurements to be $174 \pm 6 \mathrm{H}_{\mathrm{V}}$ and after 10 dpa irradiation it is $385 \pm 19 \mathrm{Hv}$ also on $304 \mathrm{SS}$ which is in reasonable agreement with the nanoindentation work here. In the literature microhardness testing was carried out on 304SS before and after cold work and irradiation and it was found that the as received material had an $\mathrm{H}_{\mathrm{V}}$ of 200 and 380 after $1.67 \mathrm{dpa}^{38}$. Additionally similar values were reported for as received material; $188 \mathrm{H}_{\mathrm{V}}$ and $270 \mathrm{H}_{\mathrm{V}}$ after $1.43 \times 10^{20}$ neutrons $/ \mathrm{cm}^{2}$ or $220 \mathrm{H}_{\mathrm{V}}$ as received and $326 \mathrm{H}_{\mathrm{V}}$ to $2.5 \mathrm{dpa}^{39}$. Therefore it can be stated that our values measured here are in reasonable good agreement with the literature.

The relationship between Hardness and Yield strength has been studied extensively and a number of different relationship parameters have been proposed. Bruemmer et $\mathrm{al}^{40}$ reported the linear relationship :

$\sigma_{\mathrm{y}}=2.5\left(\mathrm{H}_{\mathrm{V}}-68\right), \quad$ Eq. 2

while Toloczko et $\mathrm{al}^{41}$ reported 
Rodriguez et $\mathrm{al}^{31}$ proposed the equation:

$\mathrm{H}_{\mathrm{V}}=4.15 \sigma_{\mathrm{y}}$

Eq. 4

Obviously the actual calculated yield stress numbers depend on which relationship is utilized. It has to be pointed out that all of these relationships are purely empirical, and based on various materials. These relationships described above lead to a yield stress of $330 \mathrm{MPa}$ using equation 2 (Bruemmer) or $415 \mathrm{MPa}$ using equation 3 (Toloczko) 530MPa using equation 4 (Rodriguez) in the as received state. For the 10 dpa irradiated case this translates to a yield stress of $1022 \mathrm{MPa}$ applying equation 2 (Bruemmer), $1162 \mathrm{MPa}$ using equation 3 (Tolozcko) or $1216 \mathrm{MPa}$ using equation 4 (Rodriguez). Other more elaborate and theoretical based correlations do exist as proposed by J. R. Cahoon, et all ${ }^{42}$ but require strain exponent considerations which are not part of this indentation study.

J. Busby et $\mathrm{al}^{43}$ did the most elaborate empirical study on the correlation between micro harndess and yield stress with a significant number of irradiated materials and found that the change in hardness due to irradiation is related to the change in yield stress with by $\Delta \sigma_{\mathrm{y}}=3.03 \Delta \mathrm{H}_{\mathrm{V}}$. Leading to $812 \mathrm{MPa}$ difference in yield strengths (irradiated-not irradiated) based on our hardness measurements, which is slightly higher than what is calculated with the other relationships mentioned above.

Unfortunately there is not sufficient reference material available for tensile testing that would lend support for one method over another but future research will be geared towards these comparisons. It is important to note that while no further hardness change can be observed beyond $10 \mathrm{dpa}$, other phenomena like local element segregation can still exist and contribute to further materials degradation.

The second part of this work features a microstructure evaluation of the irradiated material using a micro diffraction approach. In order to evaluate the dislocation density evolution as a function of irradiation dose and thermal history, the diffraction peak width is investigated in more detail from the very edge of the sample. For statistics three diffraction patterns at each depth are selected, and from each pattern a peak within the Bragg angle range of $35^{\circ}-38^{\circ}$ is fitted with a $2 \mathrm{D}$ Gaussian function after background subtraction. The Bragg angle of the peaks is in a range but not a specific value because of two reasons. First, the areas scanned in various samples cover more than one crystal grain. Due to the characteristics of Laue diffraction technique, the Bragg angle relies on not only the d-spacing of the crystal plane, but also the orientation of the crystal grain. Thus it is not easy (or even not possible) to find a diffraction peak that appears at the same Bragg angle in all these scans. The second reason is even more universal and important. Since the synchrotron based Laue $\mu$ XRD method employed in this study provides extremely high angular orientation, even in the same crystal grain, the local crystal orientation variation due to the residual stress is detectable, indicated by the peak 
position shift. Therefore, the best option is to compare the diffraction peaks that appear in an angular range as narrow as possible. The distribution of diffraction peak FWHM along the depth direction in as-irradiated $10 \mathrm{dpa}$, irradiated $10 \mathrm{dpa}$ annealed, and as-irradiated 1 dpa is displayed in Figure 9. Comparing to the unannealed 10 dpa irradiated specimen, in which the FWHM of the diffraction peak changes from $0.5^{\circ}$ to $0.1^{\circ}$ within $4 \mu \mathrm{m}$, annealing reduces the width of the diffraction peak in the damaged layer, while the shape and width of the transition region is almost unchanged. The 1 dpa irradiated sample, in contrast, does not show as dramatic peak broadening compared to the 10dpa sample. The diffraction peak width, from a nonideal crystal with high density of paired SSDs, in the plane perpendicular to the diffraction vector is linked to the total density of dislocations (n) by ${ }^{23}$ :

$\begin{array}{lll}\mathrm{FWHM} & \propto \sqrt{n}, & \text { Eq. } 5^{44,22}\end{array}$

Therefore we can estimate the change in dislocation density by the measuring the change in FWHM. Based on the observation that the Laue diffraction peaks are broadened almost isotropically, we further assume the majority of the defects are dislocation loops, similar to the previous work shown by Jiao et al. ${ }^{37}$ Using this correlation, the dislocation density in the damaged layer in the $10 \mathrm{dpa}$ irradiated region is calculated, from Figure 9 and Eq. 5, to be 50-80 times of what it is in the non irradiated area. It is found here that annealing reduces the FWHM and therefore the assumed dislocation density by a factor of $2-4$, after $500{ }^{\circ} \mathrm{C}$ for $1 \mathrm{~h}$ but still is about 30 times higher than in the matrix. In the 1 dpa sample, the dislocation density introduced by irradiation is more than one order of magnitude less than in the 10 dpa sample.

As stated above the dislocation density is related to FWHM by equation 5. Yield strength however, is also related to the dislocation density by:

$$
\sigma_{\mathrm{y}}=\sigma_{\mathrm{y} 0}+k \cdot n^{0.5} \quad \text { Eq.6 }
$$

Therefore plotting change in hardness which is a function of yield strength vs. change in FWHM which is a function of dislocation density should lead to a linear relationship as shown in Figure 10. This correlation links the observed changes in the mechanical response with the increase in defect density that is attributed to irradiation.

\section{Conclusion}

In order to understand the microstructural evolution due to irradation damage and the resulting mechanical response, it is necessary to fully characterize materials that are utilized in irradiation environments. Since ion beam irradiation does not allow the use of large scale mechanical tests small scale mechanical testing like nanoindentation can be utilized. In this study we correlated mechanical property response that is associated with various irradiation conditions with the defects induced during irradiation. Comaring the nanoinaddentation data with FWHM from Laue micro diffraction we suggest that a linear correlation between these two paramters may exist considering that FWHM and nanoindentation is based on the dislocation density. Furhter investigation is needed on ths 
matter since only limited datapoints are aviabale at this point in time. It was found that the 304SS shows no The 1dpa sample shows a gradient throught the implnatation depth leading to the conclusion that the defect saturation is reached at 10dpa.

\section{Acknowledgements}

The ALS is supported by the Director, Office of Science, DOE-BES, Materials Science Division, under Contract No. DE-AC02-05CH11231 at LBNL. Additional support for ALS beam line 12.3.2 comes from NSF (0416243). KC acknowledges the support from the National Young 1000 Talents Program of China. We want to thank EPRI for supporting this research as well as DOE-NEUP under the award DE-FOA-0000799 


\section{Figures:}

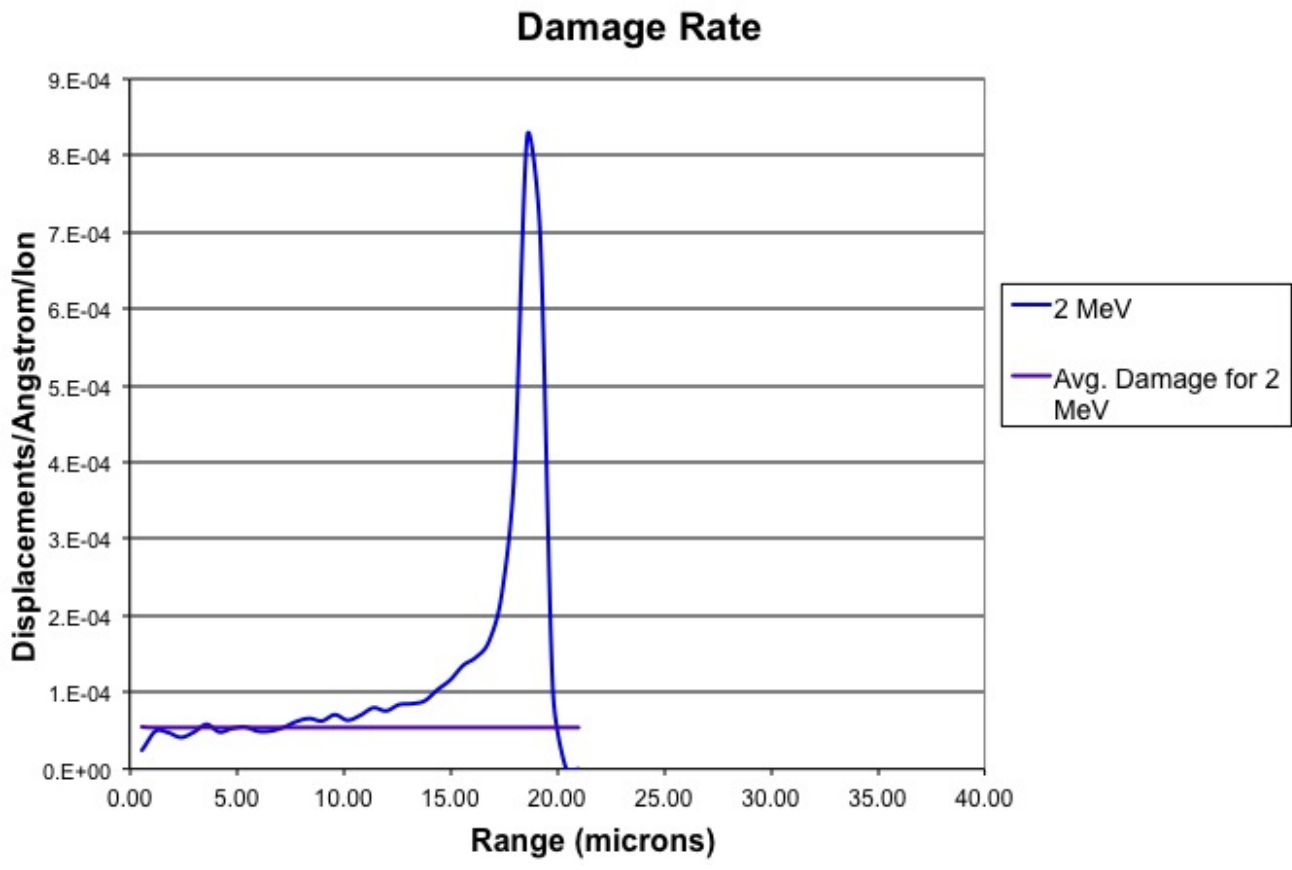

Figure 1: Calculated SRIM dose profile showing the damage rate.

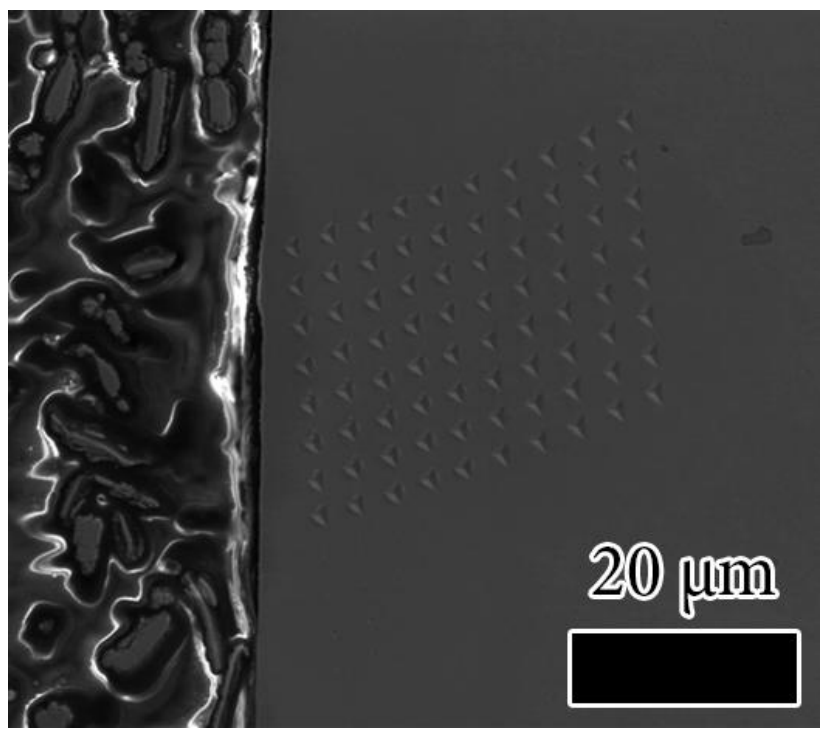

Figure 2: SEM micrograph of the nano indents in the ion irradiated region on the 10dpa sample. 


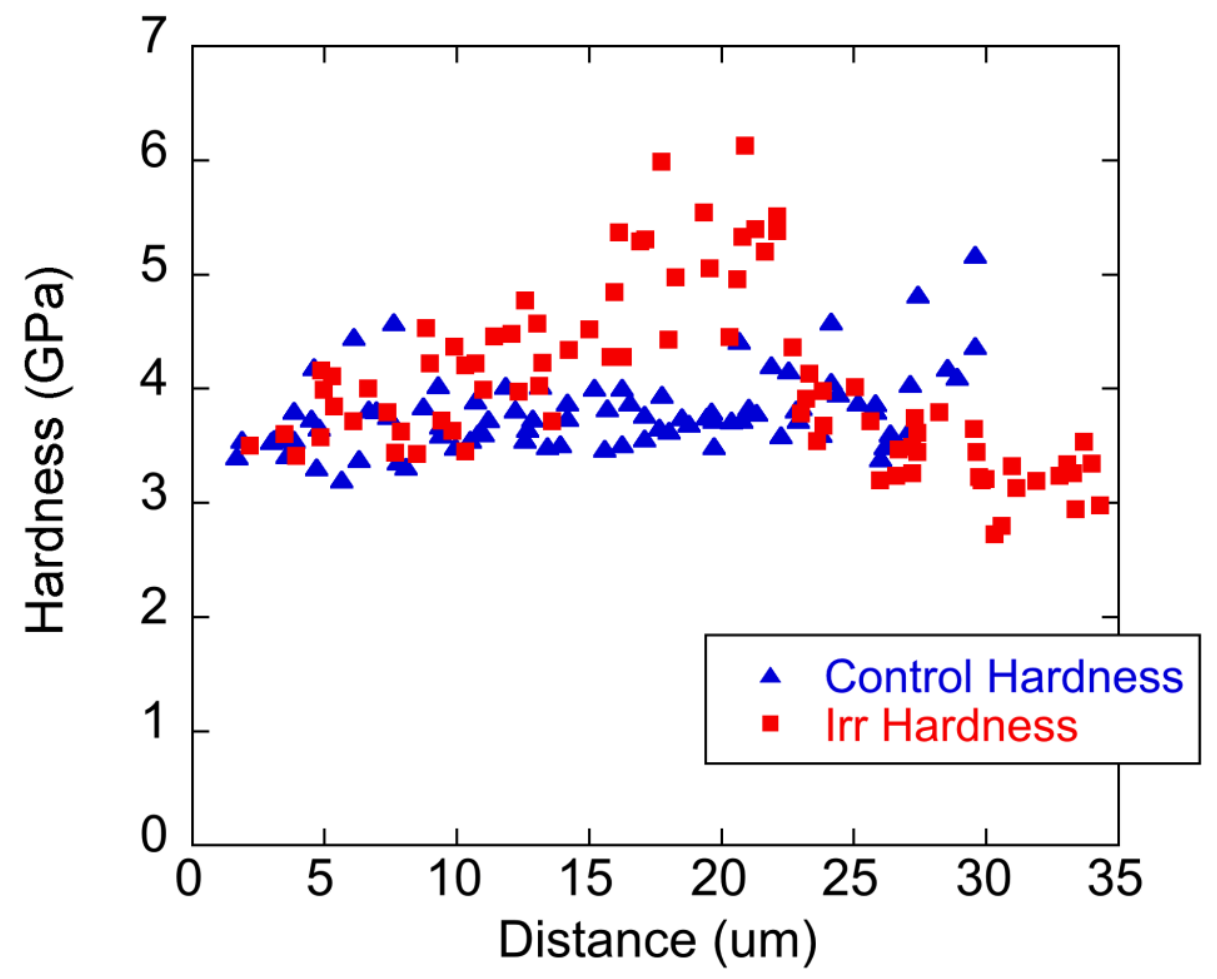

Figure 3: Nanoindentation profile for the 1 dpa irradiated 304 stainless steel specimen.

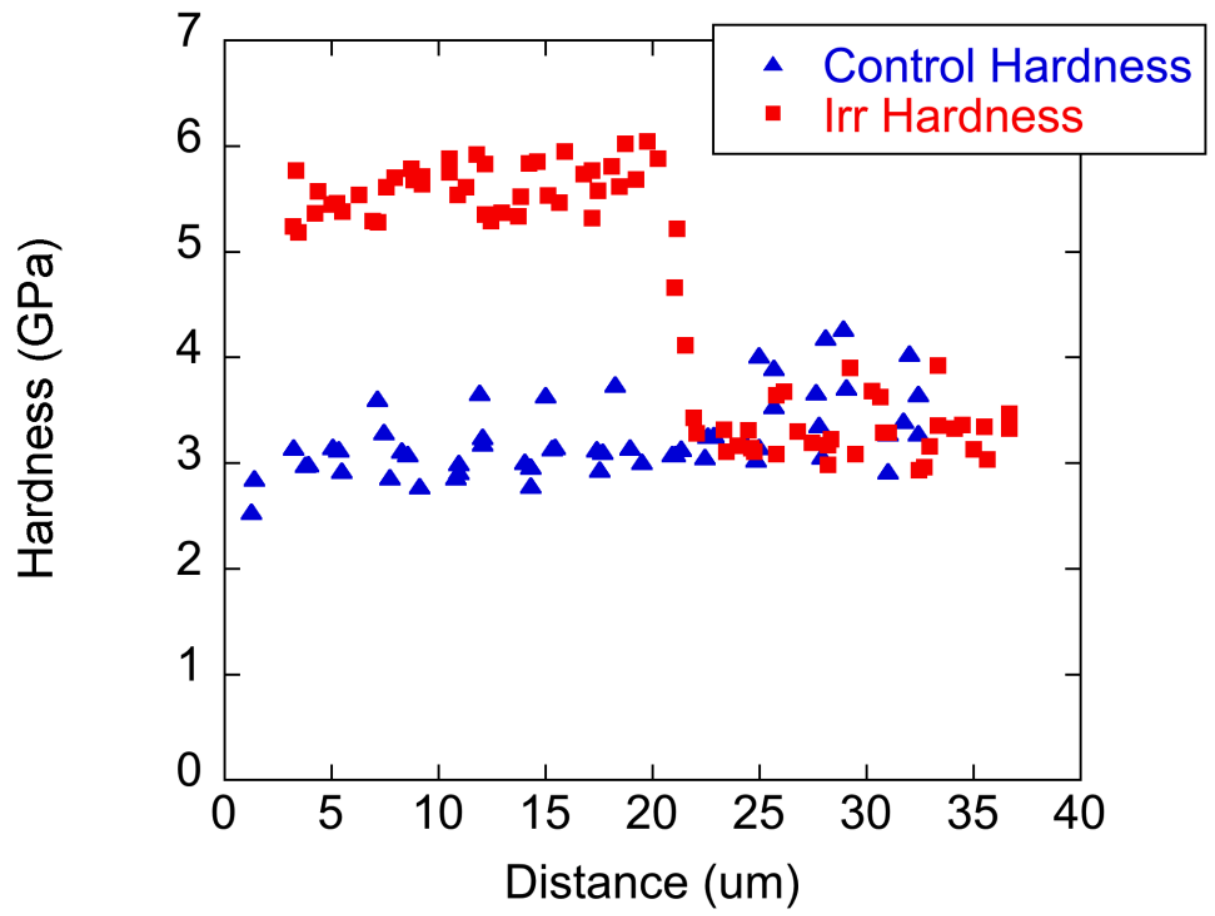

Figure 4: Nanoindentation profile for the 10 dpa irradiated 304 stainless steel specimen. 


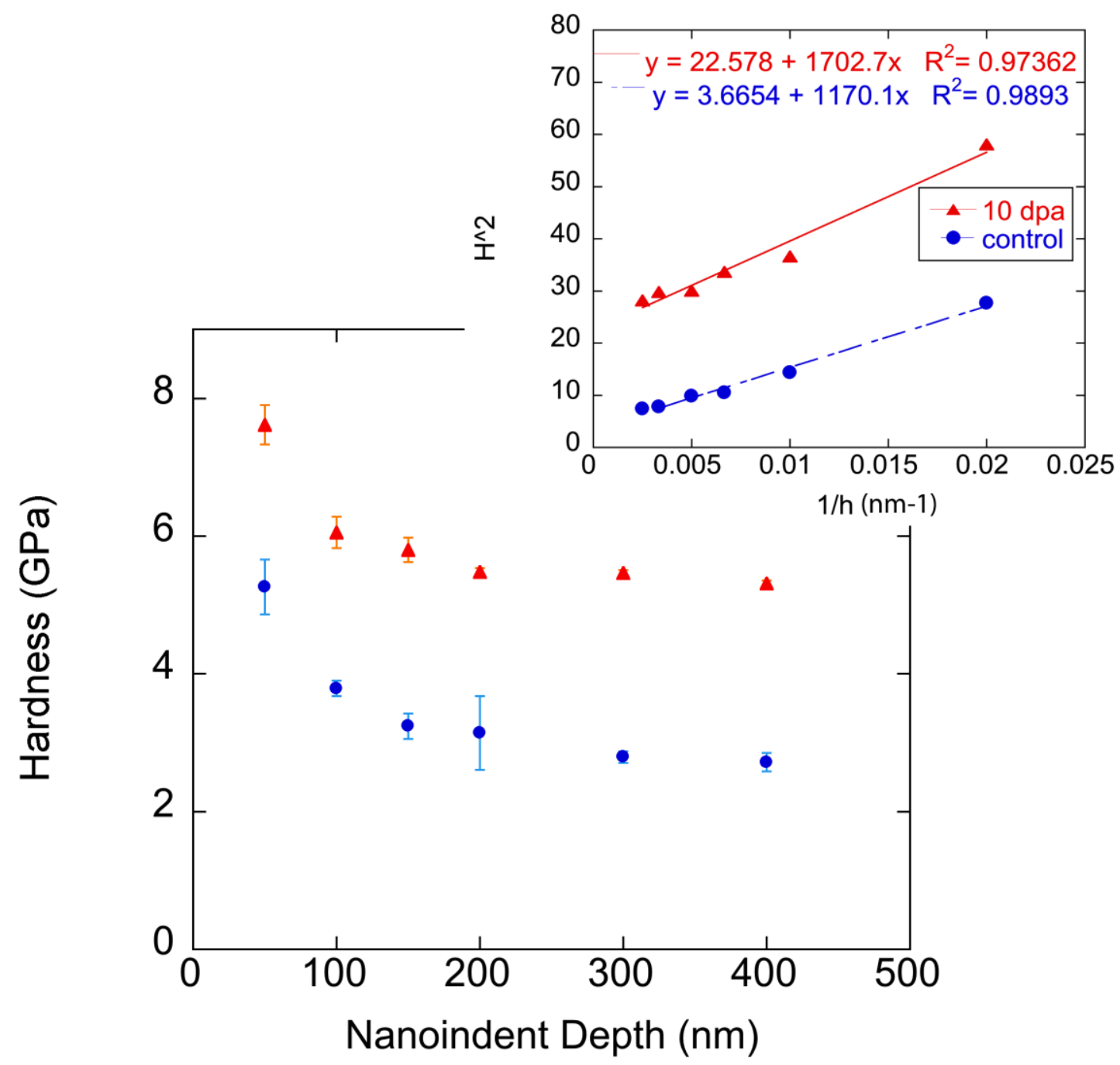

Figure 5: Plot summary of the size effect study performed both in the irradiated and control regions of the 10 dpa ion irradiated sample. 


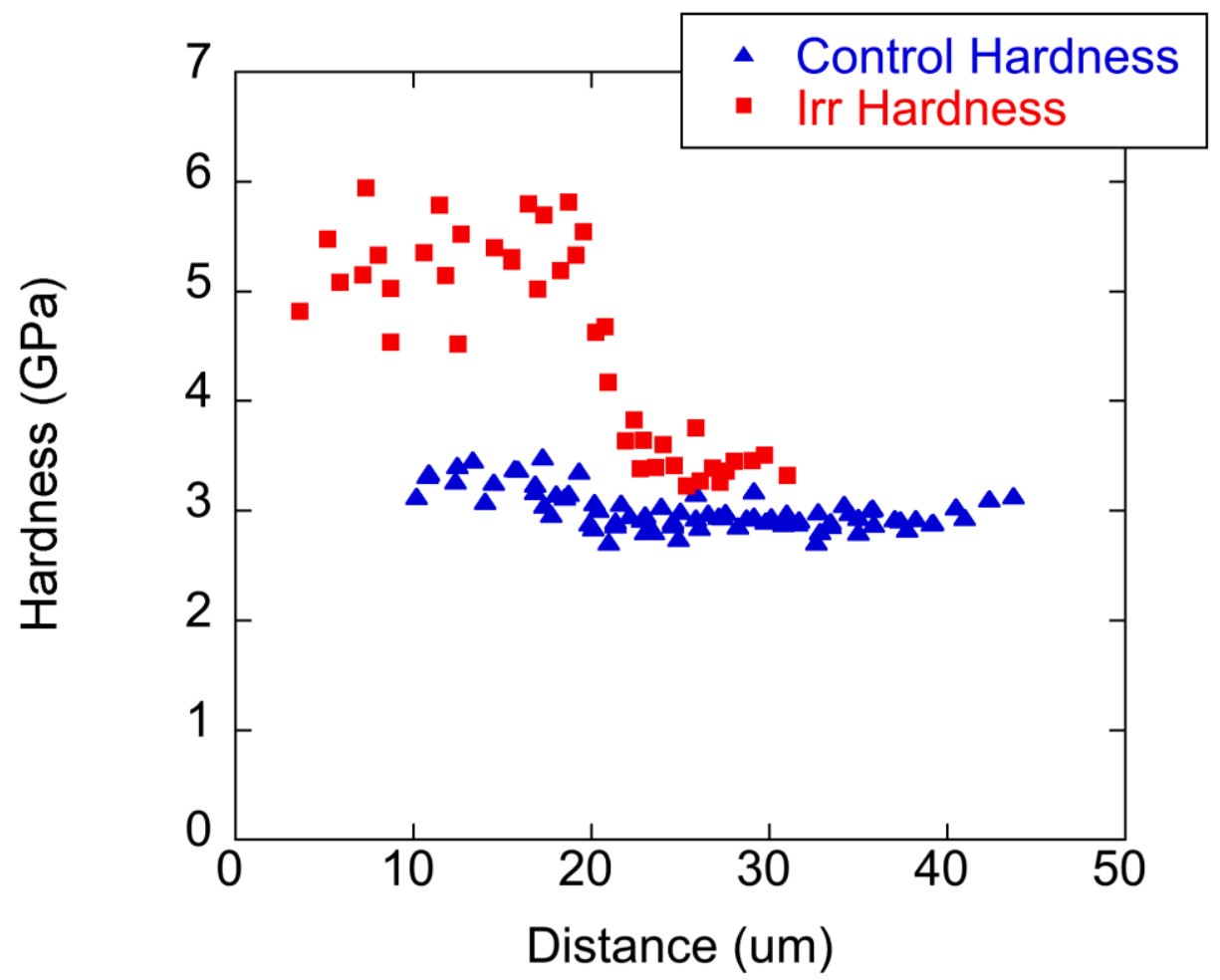

Figure 6: Nanoindentation profile for the 10 dpa irradiated stainless steel that was annealed for $1 \mathrm{~h}$ at $500{ }^{\circ} \mathrm{C}$. 

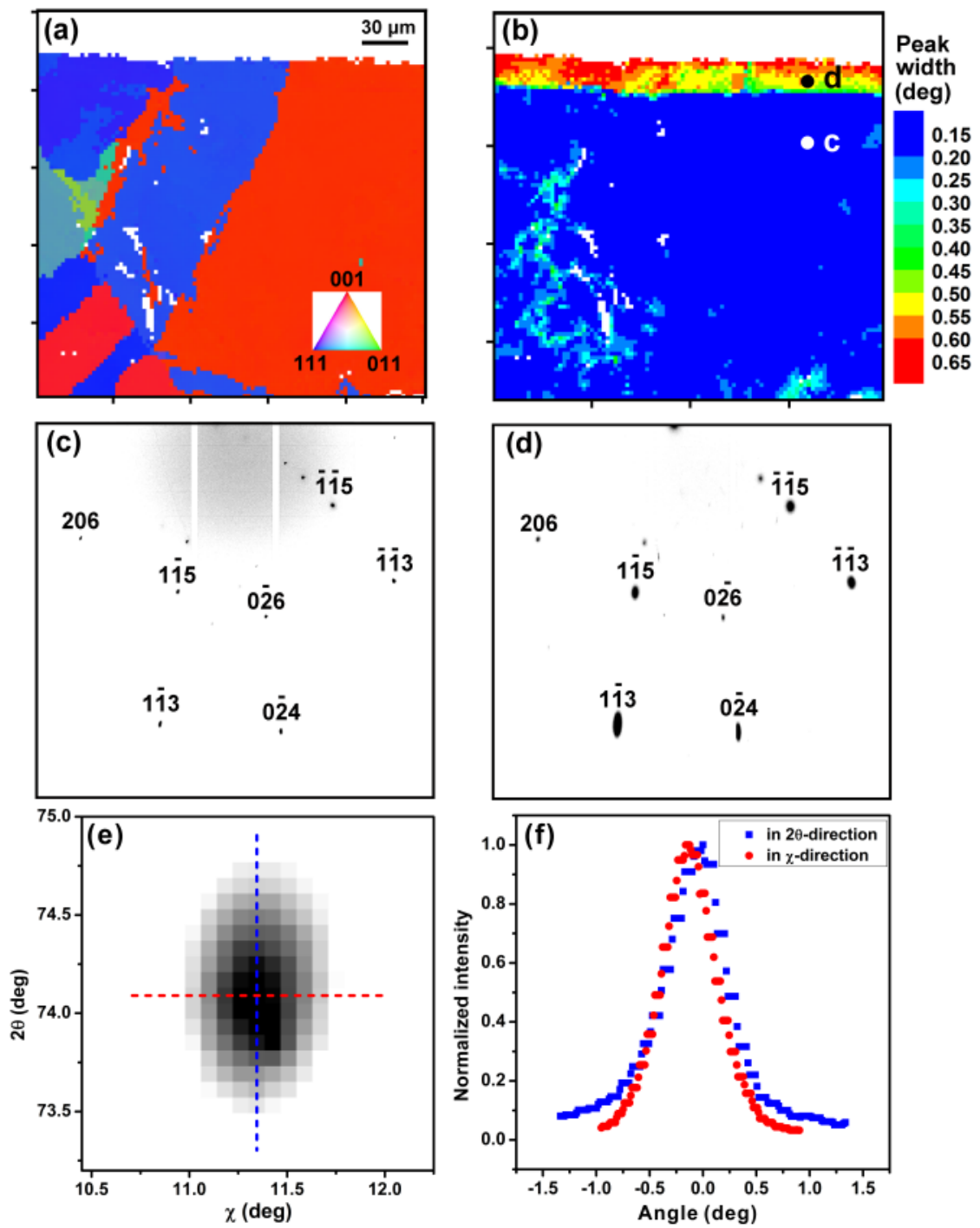

Figure 7: Orientation map of the material investigated (10dpa) a), and dislocation density map based on FWHM evaluation b). The scale bar provided applies to both images. The increase in FWHM of the irradiated area is clearly visible. Peaks observed in the unirradiated area c). Peaks observed in the irradiated area d). Peak broadening can be clearly observed. Peak $1 \overline{1} 5$ is re-plotted in the $2 \theta-\chi$ space e) and the intensity is scanned in both $2 \theta$ and $\chi$ directions $\mathrm{f}$ ), so that the peak width is measured by fitting the intensity with Gaussian distribution function. 

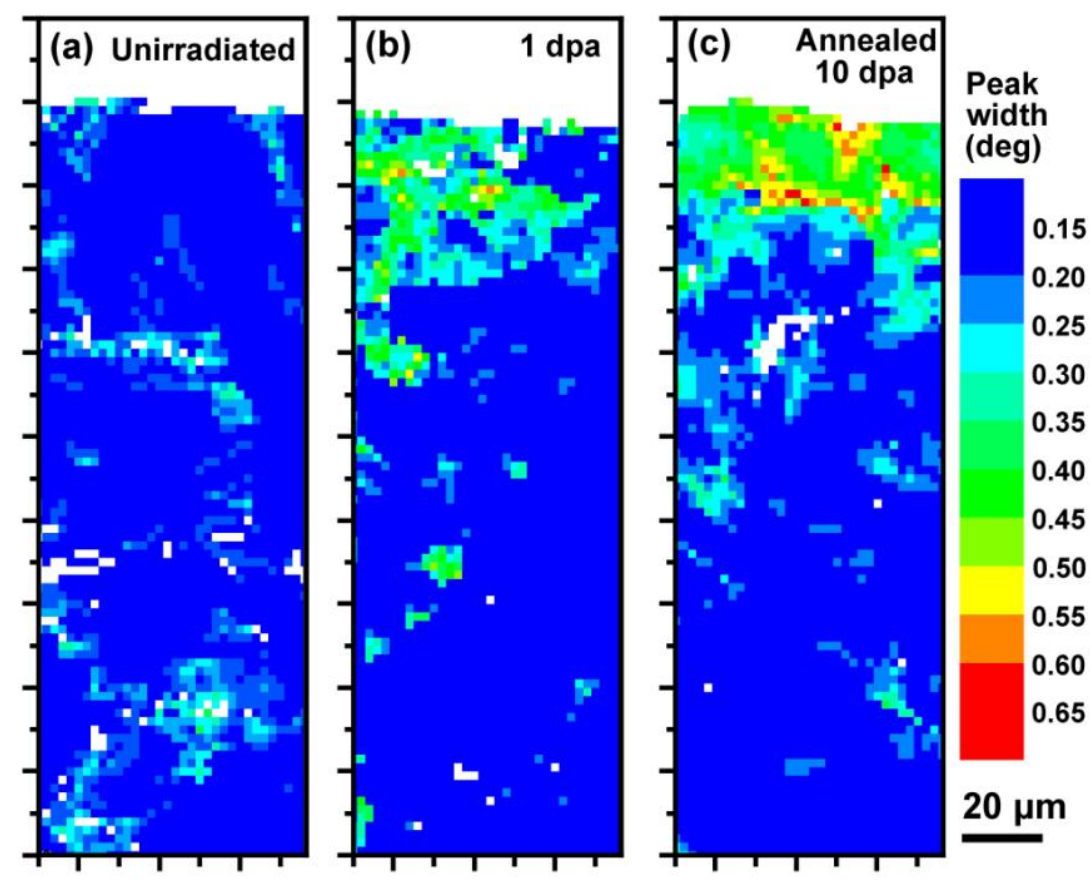

Figure 8: FWHM map of the sample unirradiated a), 1dpa irradiated b), and annealed 10dpa irradiated c) samples. The scale bar provided applies to all three images.

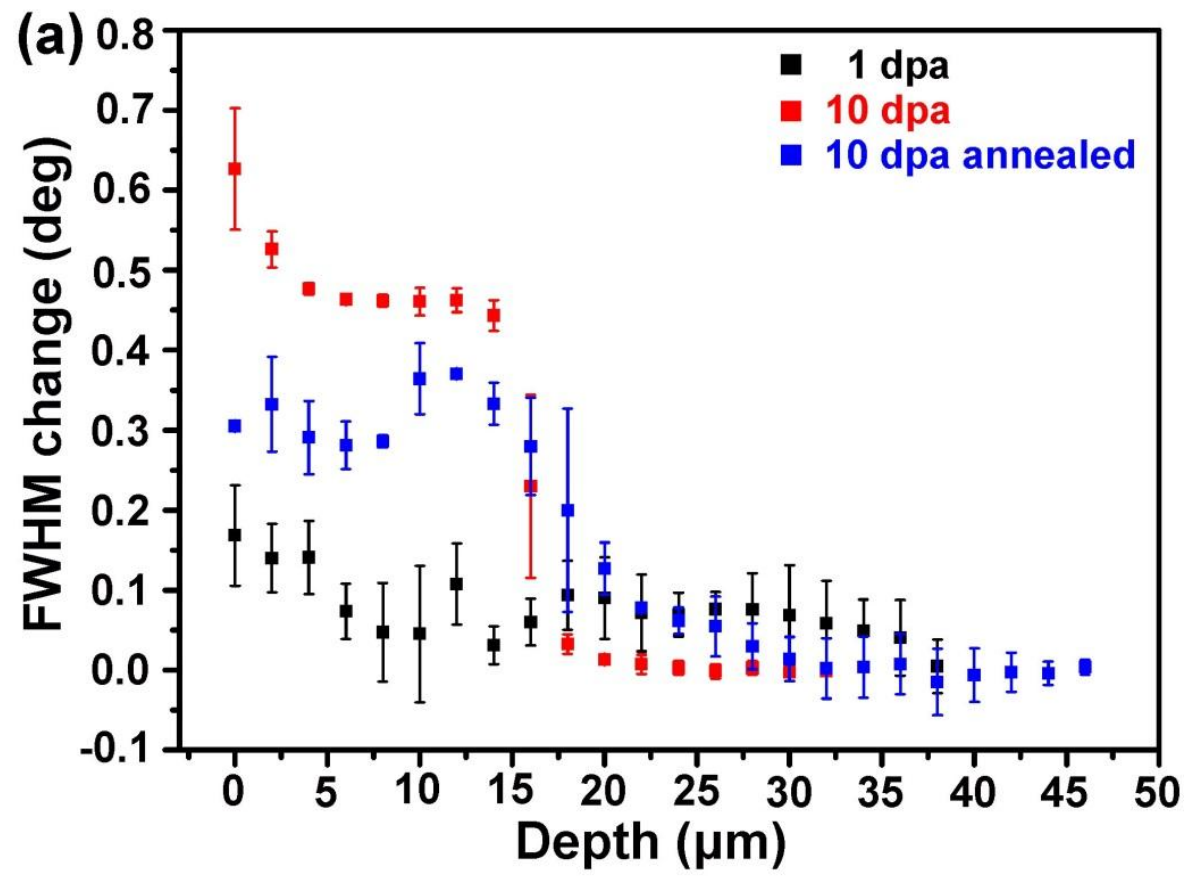

Figure 9: Plot of change in FWHM vs depth from the irradiated surface of each sample. 


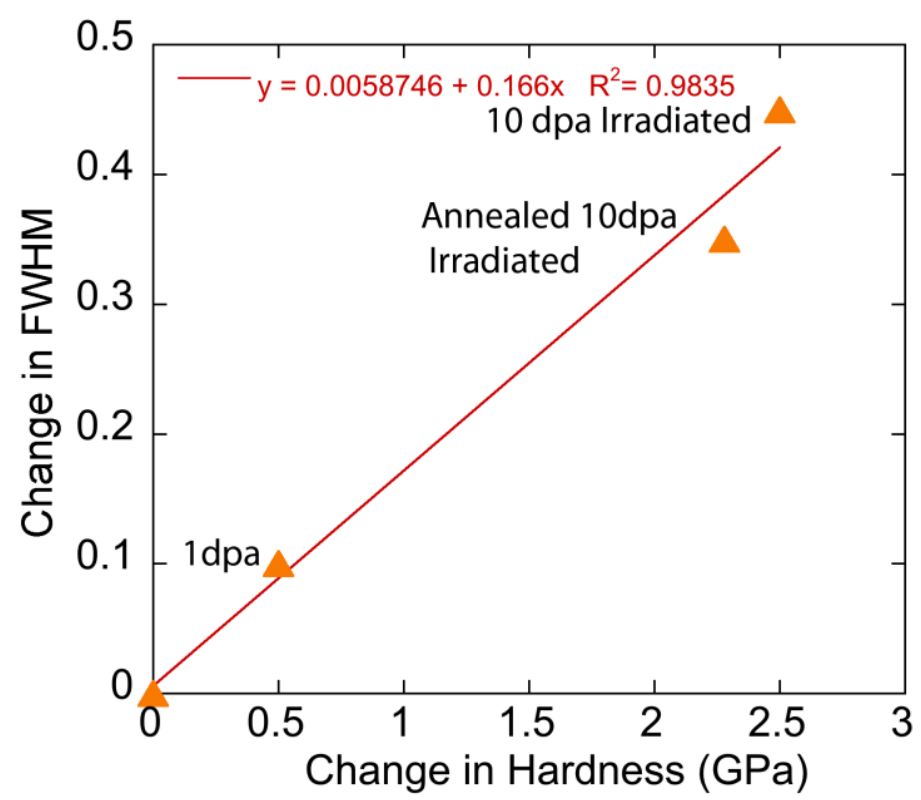

Figure 10: the change in FWHM is plotted against the change in hardness. It can be seen that the relationship is rather linear with a $R^{2}$ value of 0.9835 .

1. Kiener, D. et al. Application of small-scale testing for investigation of ion-beamirradiated materials. J. Mater. Res. 27, 2724-2736 (2012).

2. Murty, K. L. \& Charit, I. Structural materials for Gen-IV nuclear reactors: Challenges and opportunities. J. Nucl. Mater. 383, 189-195 (2008).

3. Zinkle, S. J. \& Busby, J. T. Structural materials for fission \& fusion energy. Mater. Today 12, 12-19 (2009).

4. Mansur, L. . et al. Materials needs for fusion, Generation IV fission reactors and spallation neutron sources - similarities and differences. J. Nucl. Mater. 329-333, 166-172 (2004).

5. Sencer, B. et al. Microstructural origins of radiation-induced changes in mechanical properties of $316 \mathrm{~L}$ and $304 \mathrm{~L}$ austenitic stainless steels irradiated with mixed spectra of high-energy protons and spallation neutrons. J. Nucl. Mater. 296, 112-118 (2001).

6. McMurtrey, M. D., Was, G. S., Patrick, L. \& Farkas, D. Relationship between localized strain and irradiation assisted stress corrosion cracking in an austenitic alloy. Mater. Sci. Eng. A 528, 3730-3740 (2011).

7. Sharon, J. A., Hattar, K., Boyce, B. L. \& Brewer, L. N. Compressive Properties of $\langle 110\rangle \mathrm{Cu}$ Micro-Pillars after High-Dose Self-Ion Irradiation. Mater. Res. Lett. 2, 57-62 (2014). 
8. Wirth, B. D. Materials science. How does radiation damage materials? Science 318, 923-4 (2007).

9. Azevedo, C. R. F. A review on neutron-irradiation-induced hardening of metallic components. Eng. Fail. Anal. 18, 1921-1942 (2011).

10. Was, G. S. et al. Emulation of neutron irradiation effects with protons $\square$ : validation of principle. J. Nucl. Mater. 300, 198-216 (2002).

11. Hosemann, P. et al. Nanoindentation on ion irradiated steels. J. Nucl. Mater. 389, 239-247 (2009).

12. Kiener, D., Hosemann, P., Maloy, S. A. \& Minor, A. M. In situ nanocompression testing of irradiated copper. Nat. Mater. 10, 608-13 (2011).

13. Ice, G. E., Budai, J. D. \& Pang, J. W. L. The race to x-ray microbeam and nanobeam science. Science 334, 1234-9 (2011).

14. Ziegler, J. F. \& Biersack, J. P. SRIM program. (IBM Corp, 2008).

15. Gupta, G., Jiao, Z., Ham, A. N., Busby, J. T. \& Was, G. S. Microstructural evolution of proton irradiated T91. J. Nucl. Mater. 351, 162-173 (2006).

16. Hosemann, P. et al. Mechanical characteristics of SiC coating layer in TRISO fuel particles. J. Nucl. Mater. 442, 133-142 (2013).

17. Rice, P. M. \& Stoller, R. E. The effect of solutes on defect distributions and hardening in ion-irradiated model ferritic alloys. 244, 219-226 (2008).

18. Oliver, W. \& Pharr, G. An improved technique for determining hardness and elastic-modulus using load and displacement sensing indentation experiments. $J$. Mater. Res. 7, 1564-1583 (1992).

19. Kunz, M. et al. A dedicated superbend x-ray microdiffraction beamline for materials, geo-, and environmental sciences at the advanced light source. Rev. Sci. Instrum. 80, 035108 (2009).

20. Kunz, M., Chen, K., Tamura, N. \& Wenk, H.-R. Evidence for residual elastic strain in deformed natural quartz. Am. Mineral. 94, 1059-1062 (2009).

21. Tamura, N. in Strain Dislocation Gradients from Diffr. (Barabash, R. \& Ice, G. E.) 125-155 (Imperial College Press, 2014).

22. Barabash, R. I. et al. Quantitative analysis of dislocation arrangements induced by electromigration in a passivated Al $(0.5 \mathrm{wt} \square \% \mathrm{Cu})$ interconnect. J. Appl. Phys. 93, 5701 (2003). 
23. Maaß, R. et al. Crystal rotation in $\mathrm{Cu}$ single crystal micropillars: In situ Laue and electron backscatter diffraction. Appl. Phys. Lett. 92, 071905 (2008).

24. Chen, K., Tamura, N., Valek, B. C. \& Tu, K. N. Plastic deformation in Al (Cu) interconnects stressed by electromigration and studied by synchrotron polychromatic x-ray microdiffraction. J. Appl. Phys. 104, 013513 (2008).

25. Valek, B. C. et al. Electromigration-induced plastic deformation in passivated metal lines. Appl. Phys. Lett. 81, 4168 (2002).

26. Ice, G. E. \& Barabash, R. I. in Dislocations in Solids (Nabarro, F. R. N. \& Hirth, J. P.) 499-601 (Elsevier, 2007).

27. Pokor, C., Brechet, Y., Dubuisson, P., Massoud, J.-P. \& Averty, X. Irradiation damage in 304 and 316 stainless steels: experimental investigation and modeling. Part II: Irradiation induced hardening. J. Nucl. Mater. 326, 30-37 (2004).

28. Maloy, S. A. et al. The mechanical properties of 316L/304L stainless steels, Alloy 718 and Mod 9Cr-1Mo after irradiation in a spallation environment. J. Nucl. Mater. 296, 119-128 (2001).

29. Schulz, F., Hanemann, H. \& Fuer, Z. Metall. 33, 124 (1941).

30. Zong, Z. et al. Indentation size effects in the nano- and micro-hardness of fcc single crystal metals. Mater. Sci. Eng. A 434, 178-187 (2006).

31. Rodriguez, R. \& Gutierrez, I. Correlation between nanoindentation and tensile propertiesInfluence of the indentation size effect. Mater. Sci. Eng. A 361, 377-384 (2003).

32. Chicot, D. Hardness length-scale factor to model nano- and micro-indentation size effects. Mater. Sci. Eng. A 499, 454-461 (2009).

33. Huang, Y. et al. A model of size effects in nano-indentation. J. Mech. Phys. Solids 54, 1668-1686 (2006).

34. Nix, W. D. \& Gao, H. Indentation Size Effects in Crystalline Materials: A Law for Strain Gradient Plasticity. J. Mech. Phys. Solids 46, 411-425 (1998).

35. Fischer-Cripps, A. C. Nanoindentation. 26, 269 (Springer, 2004).

36. Rice, P. M. \& Stoller, R. E. Correlation of Nanoindentation and Conventional Mechanical Property Measurements. Mater. Res. Soc. Symp. Proc. 649, q7.11.1q7.11.6 (2001). 
37. Jiao, Z., Chen, Y., Marquis, E. \& Was, G. S. Post-Irradiation Annealing in Mitigation of IASCC of Proton-Irradiated Stainless Steel. in 16th Int. Conf. Environ. Degrad. Mater. Nucl. Power Syst. Water React. 1-8 (2013).

38. Hash, M. C., Busby, J. T. \& Was, G. S. The effect of hardening source in proton irradiation-assisted stress corrosion cracking of cold worked type 304 stainless steel. Am. Soc. Test. Mater. Spec. Tech. Publ. 1447, 92-104 (2004).

39. Busby, J. T., Was, G. S. \& Kenik, E. A. Isolating the effect of radiation-induced segregation in irradiation-assisted stress corrosion cracking of austenitic stainless steels. J. Nucl. Mater. 302, 20-40 (2002).

40. Bruemmer, S. Characterization of Neutron-Irradiated 300-Series Stainless Steels to Assess Mechanisms of Irradiation Assisted Stress Corrosion Cracking . 1-104 (2006).

41. Toloczko, M., Lucas, G., Odette, G., Stoller, R. \& Hamilton, M. An investigation of microstructures and yield strengths in irradiated austenitic stainless steels using small specimen techniques. in 17th Int. Symp. Eff. Radiat. Mater. 902 (ASTM STP 1270, 1996).

42. Cahoon, J. R., Broughto, W. \& Kutzak, A. The Determination of Yield Strength From Hardness Measurements. Metall. Trans. 2, 1-5 (1979).

43. Busby, J. T., Hash, M. C. \& Was, G. S. The relationship between hardness and yield stress in irradiated austenitic and ferritic steels. J. Nucl. Mater. 336, 267-278 (2005).

44. Barabash, R. I., Ice, G. E. \& Walker, F. J. Modeling and Numerical Simulations of Microdiffraction from Deformed Crystals. Mat. Res. Soc. Symp. Proc. 731, W.8.3.1-W.8.3.7 (2002). 